\title{
TWO GENERA AND ONE SPECIES NEWLY RECORDED WITH DESCRIPTION OF FIVE NEW SPECIES OF THE SUBFAMILY MICROGASTRINAE (HYMENOPTERA: BRACONIDAE) FROM VIETNAM
}

\author{
KHUAT DANG LONG \\ Institute of Ecology \& Biological Resources (IEBR), Ha Noi, Vietnam \\ C. VAN ACHTERBERG \\ Nationaal Natuurhistorisch Museum (RMNH) Leiden, The Netherlands
}

\begin{abstract}
The genera Alloplitis Nixon, 1965 and Protapanteles Ashmead, 1898 (subgenus Nyereria Mason, 1981) (Braconidae: Microgastrinae) are newly recorded for Vietnamese fauna.

Three valid species of the genus Alloplitis Nixon, 1965 and one species of the subgenus Nyereria Mason [15] are currently recognized from the Oriental region, and in this paper one species is newly recorded for the fauna of Vietnam, Alloplitis guapo Nixon, 1965 and five new species are described and illustrated from Vietnam, Alloplitis albiventris Long \& van Achterberg, sp. n., A. laevigaster Long \& van Achterberg, sp. n., A. vietnamicus Long \& van Achterberg, sp. n., Protapanteles (Nyereria) albicentrus Long \& van Achterberg, sp. n. and P. (Nyereria) yenthuyensis Long \& van Achterberg, sp. n.. In collections these genera are rare, probably because Braconidae has been only limited collected by Malaise traps in Vietnam.

For recognition of the subfamily Microgastrinae, see Nixon (1965) [10], for division of groups of the genus Apanteles s. 1. see Wilkinson (1932) and Nixon $(1965,1967)$, for reclassification of the subfamily Microgastrinae see Nixon (1965) and Mason (1981). For the terminology used in this paper, see van Achterberg (1993). The scale-lines of the plates indicate $1 \mathrm{~mm}$.
\end{abstract}

Key words: Braconidae, Microgastrinae, Alloplitis, Nyereria, Protapanteles, new species, Vietnam.

\section{TAXONOMY}

\section{Key to Vietnamese species of the genus Alloptilis Nixon}

1. Tergite 3 rugose and much shorter than tergite 2 (about half as long as tergite 2), fore wing with small second submarginal cell, hind coxa yellow $\square$

A. guapo Nixon, 1965

- Tergite 3 smooth and longer than $2 / 3$ of tergite 2 , if distinctly shorter than half of tergite 2 then tergite 2 smooth or hind coxa black or blackish brown, fore wing with large or medium-sized second submarginal cell

2. Tergite 3 less than half as long as tergite 2 , tergites $2+3$ smooth, inner hind tibial spur 0.5 times as long as hind basitarsus

A. laevigaster sp. $\mathrm{n}$.

- Tergite 3 about as long as tergite 2, tergite 2 rugose, inner hind tibial spur shorter or as long as

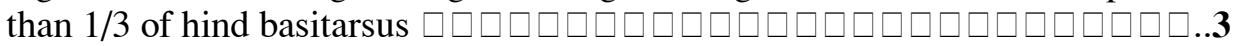

3. Fore wing with a large second submarginal cell, hind femur and tibia yellow, metasoma black or blackish brown

A. vietnamicus sp. $\mathrm{n}$.

- Fore wing with a medium-sized second submarginal cell, hind femur and tibia black, metasoma brown but ventrally white or whitish yellow A. albiventris sp. $\mathrm{n}$. 


\section{Alloplitis albiventris Long \& van Achterberg, sp. n. (figs 1-8)}

Material. Holotype, , (IEBR), Mic.570, Vietnam: Hoabinh (Yenthuy), fruit orchard (MT), 20 23'47N 105 36'14E, 01-10.IV.2002, K. D. Long.

Holotype, , , body length $2.7 \mathrm{~mm}$, fore wing length $2.7 \mathrm{~mm}$, antenna $3.0 \mathrm{~mm}$.

Head (figs 1-2).- Antenna longer than body, length of third segment as long as fourth segment; conical apical segment as long as penultimate segment; $12^{\text {th }}-16^{\text {th }}$ antennal segments distinctly swollen (fig. 6). Head in frontal view with antennal sockets far above middle level of eyes (fig. 2); frons with prominent long median carina and convergent rugosities (V-shaped); width of face 0.7 times height of eye and clypeus combined (15: 21); height of eyes 2.4 times width (22: 9); tentorial pits large, distance between tentorial pits 3.0 times distance from pit to eye margin (9: 3 ). Head in dorsal view with ocelli small and in a low triangle, the anterior tangent to posterior ocelli cutting anterior ocellus (fig. 1); POL 1.5 times OOL and 2.0 times diameter of posterior ocellus (OD) (6: 4: 3). Face dull, rugosepunctate with a long median smooth carina (fig. 2) shiny; vertex, occiput and temple close rugose-punctate.
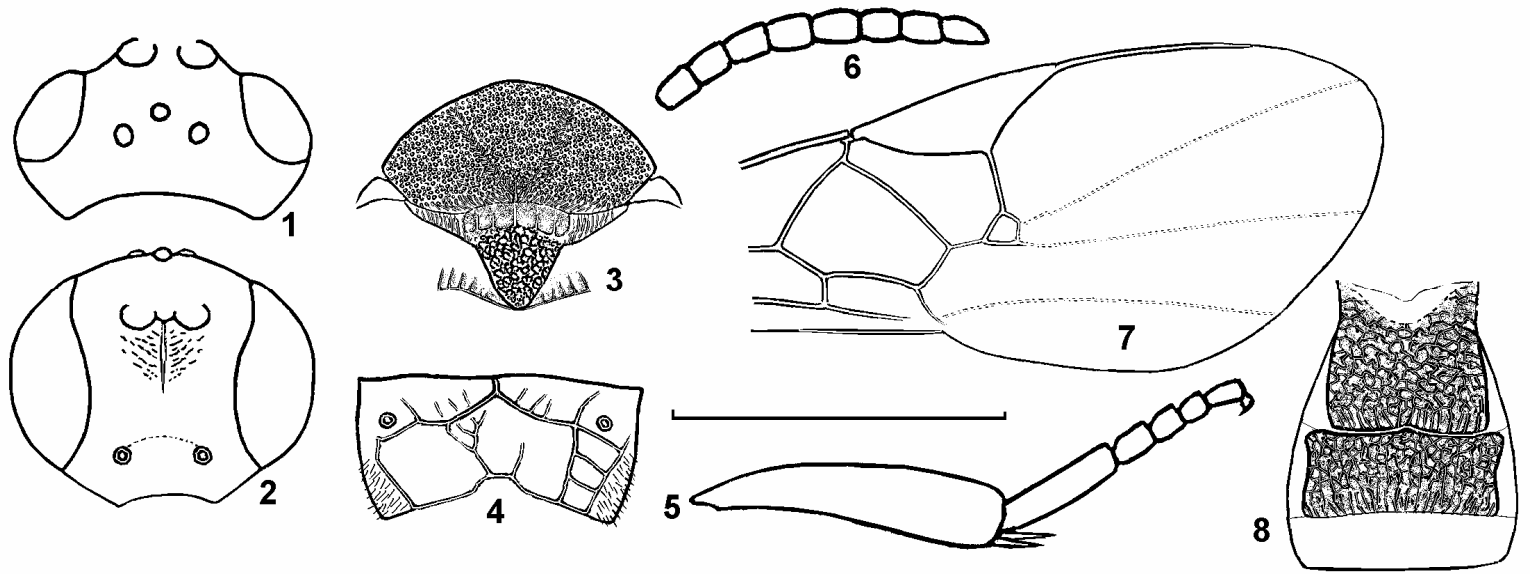

Figs 1-8. Alloplitis albiventris Long \& van Achterberg, sp. n.

1. head, dorsal aspect; 2 . head, frontal aspect; 3 . mesonotum; 4. propodeum;

5. hind leg; 6 . fore wing; 7 . apical antennal segments.

Mesosoma.- Mesonotum as wide as head in dorsal view (36: 32); mesoscutum close reticulate-punctate with longitudinal rugosities posteriorly; scutellum reticulate-punctate; scutellar sulcus deep and wide with 5 carinae and 0.3 times as long as scutellum (4: 12) (fig. $3)$; mesopleuron rugose-punctate anteriorly, shiny and smooth posteriorly; mesosternum rugose-punctate anteriorly and rugose posteriorly; precoxal sulcus wide, shallow with reticulate punctures; propodeum with lateral carinae, irregular costulae and areolate-rugose.

Wings.- Fore wing with radial vein $r$ arising just after middle of pterostigma; vein $r$ twice long as vein 2-SR (8: 4), and little longer than width of pterostigma (8: 7) (fig. 6); metacarp longer than length of pterostigma (21: 20); vein 1-CU1 0.6 times as long as vein 2-CU1 (6: 10), 2-CU1 1.25 times as long as vein $\mathrm{m}$-cu.

Legs.- Hind tibia gradually swollen apically (fig. 5) and about 1.1 times as long as hind tarsus 1-5 (35: 33); inner hind tibial spurs about 0.5 times hind basitarsus (7: 15); fourth tarsal segment shorter than fifth tarsal segment $(3: 4)$ (fig. 5).

Metasoma.- Length of horizontal surface of tergite 1 about 0.6 times as long as its apical width; third tergite 0.8 times as long as second tergite (8: 10) (fig. 8); surface of tergite $1+2$ areolate-rugose; second metasomal suture and third tergite smooth. 
Colour.- Body black; palpi white; scape yellow, light brown ventrally with long setae; flagellum yellow, darker apically; fore and middle legs yellow, hind legs black except trochanters and tarsus 2-5 yellow; hind tibial spurs white.

Male: unknown.

Notes: Alloplitis albiventris sp. $\mathrm{n}$. is close to A. guapo Nixon, 1965, described from Philippines, but it differs from the latter by the following features: i)- tergite 3 smooth and about as long as tergite 2 (0.9 times), ii)propodeum with lateral carinae, iii)- frons with prominent carina and convergent rugosities (Vshaped) and iiii)- metasoma white or whitish yellow ventrally.

\section{Alloplitis laevigaster Long \& van Achterberg, sp. n. (figs 9-16)}

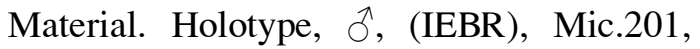
Vietnam: Hoabinh (Yenthuy), fruit orchard (MT), 20 23'47N 105'36'14E, 20-30.VII.2002, K. D. Long.

Holotype, $\widehat{\text { Oे }}$, body length $2.9 \mathrm{~mm}$, fore wing length $3.2 \mathrm{~mm}$, antenna $4.2 \mathrm{~mm}$.

Head (figs 9, 10).- Antenna longer than body, length of third segment as long as fourth segment; apical segment a little longer than penultimate segment (fig. 16). Head in frontal view with antennal sockets above middle level of eyes (fig. 10); frons with a short median carina; width of face 0.8 times height of eye and clypeus combined (19: 23); height of eyes about 2.2 times width (24: 11); tentorial pits large, distance between tentorial pits 3.3 times distance from pit to eye margin (10: 3 ). Head in dorsal view with temple roundly narrowed posteriorly (fig. 9); ocelli small and in a very low triangle, the anterior tangent to the posterior ocelli cutting anterior ocellus; POL 2.0 times OOL and 4.0 times diameter of posterior ocellus (OD) (4: 2: 1). Face finely punctate; vertex, occiput and temple rugose-punctate.

Mesosoma.- Mesonotum wider than head in dorsal view (40: 38); mesoscutum reticulaterugose, notauli present, meeting posteriorly; lateral lobes of scutum with more or less discrete punctures apically; scutellar sulcus narrow and deep, with 3 carina, about 0.3 times as long as scutellum; scutellum rugose-punctate (fig. 12); mesopleuron rugose anteriorly, polished smooth posteriorly; precoxal sulcus shallow, shiny and smooth; mesosternum punctate throughout; propodeum dull with areola and costulae (fig. 11).
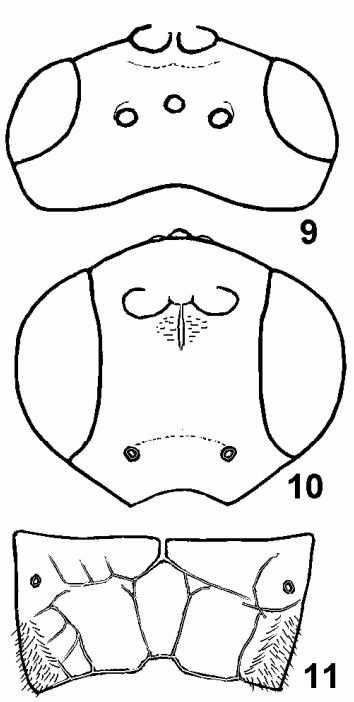

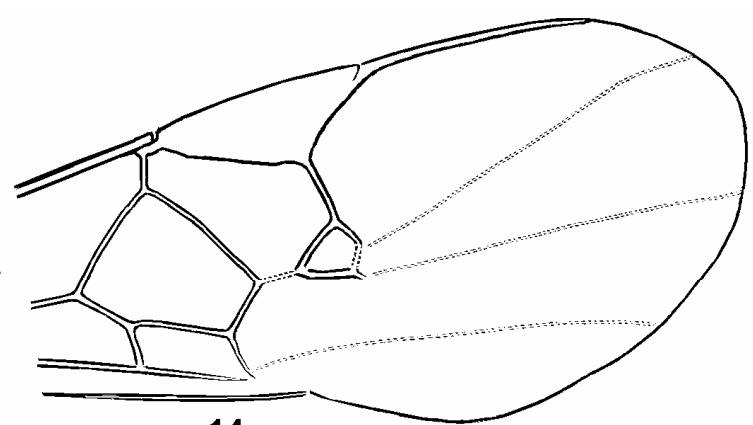

14

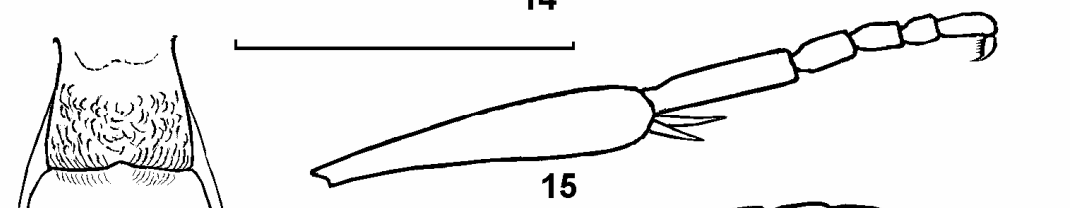

13

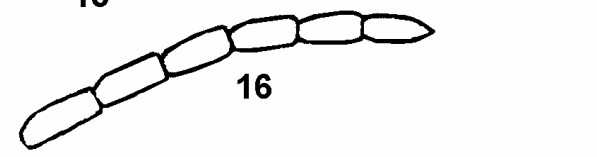

Figs 9-16. Alloplitis laevigaster Long \& van Achterberg, sp. n.

9. head, dorsal aspect; 10. head, frontal aspect; 11. propodeum; 12. mesonotum;

13. first-third tergites; 15 . hind tibia and tarsus; 16. apical antennal segments 
Wings.- Fore wing with radial vein $r$ arising at middle of pterostigma; vein $r$ as long as vein 2-SR (8: 8), and shorter than width of pterostigma (8: 9); metacarp about 1.1 times length of pterostigma (26: 23$)$; ratio of vein 1CU1: 2-CU1; $m-c u=6: 11: 7$ (fig. 14). Hind wing: cu-a curved apicad.

Legs.- Hind tibia gradually swollen apically (fig. 15); hind femur shorter than hind tibia (32: 38); hind tibia as long as hind tarsus 1-5 and more than 2.2 times as long as hind basitarsus; inner hind tibial spurs much shorter than half of hind basitarsus; fourth tarsal segment shorter fifth tarsal segment (4: 5) (fig. 15).

Metasoma.- Horizontal suface of tergite 1 as long as tergite 2 and about 0.6 times as long as apical width of tergite 1 ; third tergite 0.8 times as long as tergite 2 (8: 10); tergite 1 finely rugose, tergites $2+3$ polished smooth; second metasomal suture weak (fig. 13).

Colour.- Body black; palpi white; scape and pedicel yellow; flagellum brown, yellowish brown apically; fore and middle legs yellow; hind coxa, femur and tibia dark brown, except hind tibia whitish yellow basally; hind tarsus
$4+5$ yellow; fore wing with dense and long setae.

Female: unknown.

Notes: Alloplitis laevigaster sp.n. is close to A. completus Mason, 1981, from Malaysia but it differs from the latter by the following features: i)- metasoma black ventrally, ii)- tergite 2 smooth and iii)- tergite 3 shorter than 0.5 times tergite 2. This species is also close to A. typhon Nixon, 1965, from the Philippines but differs from the later by: i)- tergite 3 shorter than 0.5 times tergite 2, ii)- hind femur blackish brown to black.

\section{Alloplitis vietnamicus Long \& van Achterberg, sp. n. (figs 17-24)}

Material.- Holotype, क,(IEBR) "Mic.939, Vietnam: Vinhphuc (Melinh), secondary forest (MT), 21 23'N 105 42E, 20-30.III.2001, K. D. Long". Paratypes, +,,(IEBR) Mic.939a, same data as holotype; + , (RMNH), Mic.953, VN: Hagiang (Vi Xuyen), forest $700 \mathrm{~m}, \mathrm{~K}$. D. Long.

Holotype, + , body length $2.2 \mathrm{~mm}$, fore wing length $2.5 \mathrm{~mm}$, antenna $2.4 \mathrm{~mm}$.

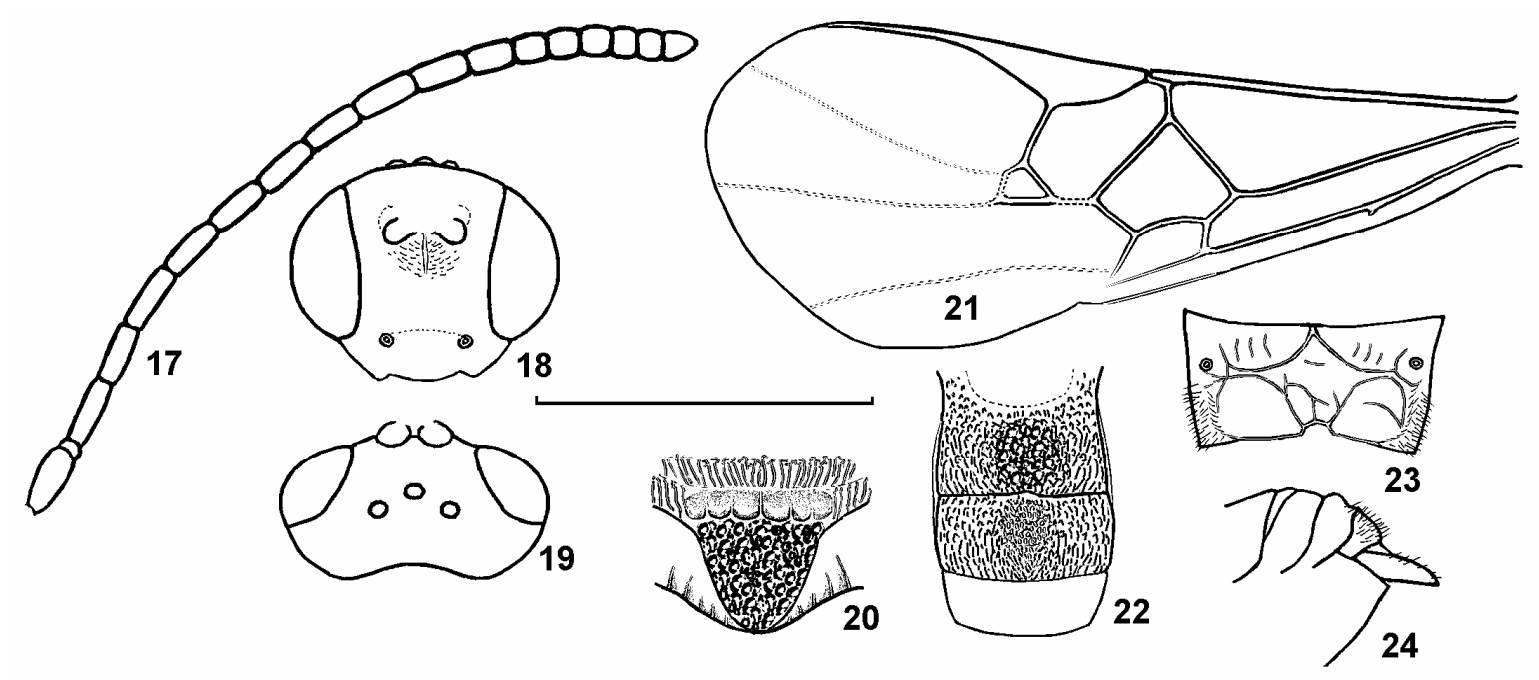

Figs 17-24. Alloplitis vietnamicus Long \& van Achterberg, sp. n.

17. antennae; 18. head, dorsal aspect; 19 . head, frontal aspect; 20 . scutellar sulcus and scutellum; 21. fore wing; 22. first-third tergites; 23. propodeum; 24. apical metasomal segments, lateral aspect.

Head (figs 18, 19).- Antennae little longer than body, penultimate segment wider than long; head in frontal view with antennal sockets above middle level of eyes (fig. 18); width of face 0.7 times height of eye and clypeus combined (15: 21); height of eyes 2.25 times width (18: 8); tentorial pits large and closed to mandible base, distance between tentorial pits 
4.0 times distance from pit to eye margin (8: 2). Head in dorsal view with temple roundly narrowed posteriorly (fig. 19); ocelli in a low triangle with posterior ocellus larger, the anterior tangent to the posterior ocelli cutting anterior ocellus; POL 1.25 times OOL and about 1.7 times diameter of posterior ocellus (OD) (5: 4: 3) (fig. 19); frons with a short longitudinal carina (fig. 18); face rugose; vertex, occiput and temple rugose.

Mesoma.- Mesonotum wider than head in dorsal view (35: 31); scutellar sulcus deep with median carina (fig. 20), about 0.4 times as long as scutellum (4: 11); mesoscutum reticulaterugose becoming striate-rugose apically; scutellum irregularly rugose; large area of mesopleuron rugose, smooth smaller area in middle; precoxal sulcus deep and rugose; mesosternum rugose; propodeum with areola and costulae, sparsely rugose (fig. 23).

Wings.- Fore wing with radial vein $r$ arising after middle of pterostigma; vein r 1.2 times as long as vein 2-SR (6: 5), and equal pterostigma; metacarp shorter than length of pterostigma (15: 20 ); ratio of vein $1-\mathrm{CU} 1: 2-\mathrm{CU} 1: \mathrm{m}-\mathrm{cu}=5: 10$ : 6 (fig. 21).

Legs.- Hind tibia gradually swollen apically; hind femur about 0.8 times hind tibia (25: 32) and shorter hind tarsus 1-5 (32: 33); hind basitarsus 0.45 times as long as hind tarsus 1-5; inner hind tibial spurs 0.4 times as long as hind basitarsus (6: 15); fourth tarsal segment shorter fifth tarsal segment (4:5).

Metasoma.- Horizontal surface of first tergite about 0.5 times as long as apical width, third tergite 0.9 times tergite 2 (9: 10) (fig. 22); tergite 1 areolate-punctate, striate laterally; tergite 2 finely punctate and striate basally; tergite 3 finely striate; ovipositor sheath projecting behind (fig. 24).

Colour.- Body black; palpi white; scape and pedicel yellow; flagellum yellow, darker apically; fore and middle legs yellow; hind legs yellow except hind coxa yellowish brown, hind tibia and basitarsus pale brown apically.

Male: unknown.

Notes: Alloplitis vietnamicus sp. n. is close to A. guapo Nixon, 1965, from Philippines but it differs from the latter by the following features: i)- tergite 3 smooth and longer than haft of tergite 2, ii)- penultimate antennal segment shorter than wide, iii)- inner hind spur 0.3 times as long as hind basitarsus and iiii)- propodeum without medial longitudinal carina.

\section{Alloplitis guapo Nixon, 1965}

This species has been found in Cucphuong national park, North Vietnam (IEBR: Ninhbinh province), MT, 20 23'06N 105 34'11E, 2030.VI.2002, K. D. Long.

\section{Key to Vietnamese species of the genus Protapanteles Ashmead, 1898 (subgenus Nyereria Mason, 1981)}

1. Ocelli in a very low triangle, anterior tangent to posterior ocelli cutting center of anterior ocellus; scutellar sulcus with 7 distinct carinae; scutellum and metanotum in dorsal view with an dorsal spine (fig. 38); a raised central area of tergite 2 with longitudinal striae, lateral area of tergite 2 and basal area of tergite 3 with sparse sharp pits; tergite 1 and hind coxa whitish yellow (but hind coxa of male reddish yellow). Protapanteles (Nyereria) albicentrus sp. $\mathbf{n}$.

- Ocelli higher, anterior tangent to posterior ocelli just touching anterior ocellus; scutellar sulcus without carinae; scutellum and metanotum in dorsal view without a strong apical spine; a raised central area of tergite and 2 tergite 3 polished smooth; tergite 1 black; and hind coxa black, pale brown apically..... Protapanteles (Nyereria) yenthuyensis sp. $n$.

\section{Protapanteles (Nyereria) albicentrus} Long \& van Achterberg, sp. n. (figs 25-32)

Material. Holotype, + , (IEBR), Apan.740, Vietnam: Hatinh (Huongson), forest, (MT), $20^{\circ} 59^{\prime} \mathrm{N} 105^{\circ} 55^{\prime} \mathrm{E}, 22 . \mathrm{VI}-08 . \mathrm{VII} .2001$, AMNH, 82
K. Long. Paratypes $\widehat{\jmath}$,(IEBR), Mic.1044, same locality but forest $900 \mathrm{~m}$, MT 05.V.1998, AMNH, K. Long; + , (RMNH) Mic.1023: Vietnam: Hatinh (Huongson), forest, (MT), $18^{\circ} 22^{\prime} \mathrm{N}$ 106 13’E, 20-28.IV.1998, AMNH, K. Long. 
Holotype, , , body length $3.4 \mathrm{~mm}$, fore wing length $3.6 \mathrm{~mm}$, antenna $3.7 \mathrm{~mm}$.

Head.- Antenna longer than body; length of third segment a little longer fourth (12: 11); apical segment equal to penultimate segment. Head in frontal view with antennal sockets just above middle level of eyes (fig. 26); width of face equal to height of eye and clypeus combined; height of eyes 0.9 times width (17: 18 ); tentorial pits small, distance between tentorial pits 1.4 times distance from pit to eye margin (7: 5); head in dorsal view with temple roundly narrowed posteriorly (fig. 25); ocelli in a very low triangle, the anterior tangent to posterior ocelli cutting anterior ocellus (fig. 25); POL 1.5 times OOL (6: 4) and 2.0 times OD (6: 3 ) (fig. 25); face punctate; vertex, occiput and temple rugose-punctate.
Metasoma.- Tergite 1 parallel-sided (fig. 30 ), length of first tergite 1.25 times apical width (15: 12); horizontal surface of first tergite shorter than apical width (9: 12); tergite 2 shorter than tergite 3 (10: 12); ovipositor sheath short and down curved apically (fig. 28).

Colour.- Head and thorax black; palpi white; scape and pedicel yellow; basal third of flagellum yellowish brown, apical two thirds of flagellum brown; fore and middle legs yellow; hind legs yellow, except apical half of hind tibia and complete hind tarsus blackish brown; wing veins yellow, pterostigma and metacarp brown; tergite 1 and sternites 1-4 white or whitish yellow; tergites $2+3$ blackish brown, tergites $4-7$ yellowish brown.

Male: unknown.

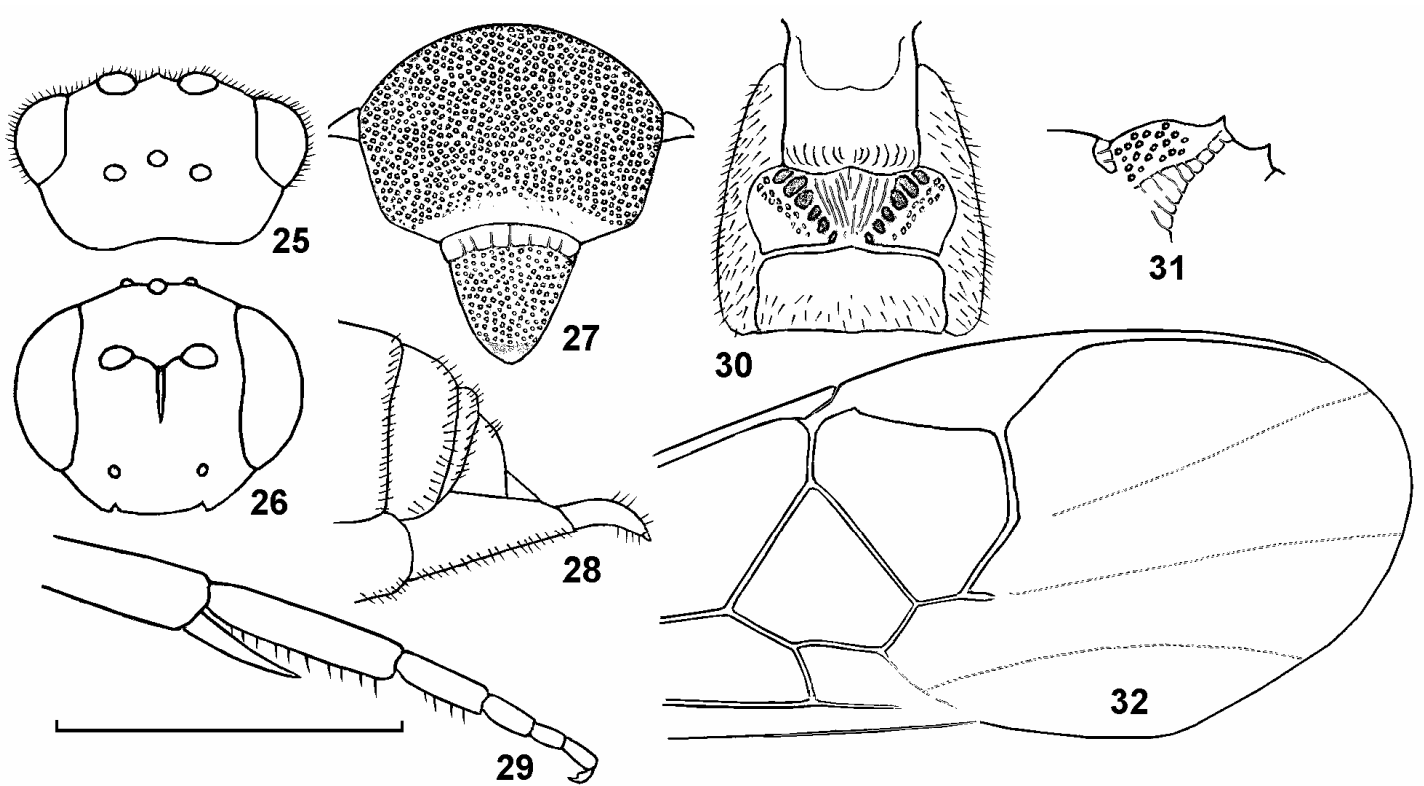

Figs. 25-32. Protapanteles (Nyereria) albicentrus Long \& van Achterberg, sp. n.

25. head, dorsal aspect; 26. head, frontal aspect; 27. mesonotum; 28. apical metasomal segments, lateral aspect; 29. hind tarsus; 30. first-third tergites; 31. scutellum and metanotum, lateral aspect; 32. fore wing.

Protapanteles (Nyereria) yenthuyensis Long \& van Achterberg, sp. n. (figs 33-39)

Material. Holotype, , (IEBR), Mic.918, Vietnam: Hoabinh (Yenthuy), (MT), forest, $2028^{\prime} \mathrm{N} 105^{\circ} 34^{\prime} \mathrm{E}, 20-30 . X .2003$, K. D. Long.

Holotype, $q$, body length $2.3 \mathrm{~mm}$, fore wing length $2.6 \mathrm{~mm}$, antenna $2.5 \mathrm{~mm}$.
Head (figs 33, 34)- Antenna distinctly longer than body, length of third segment slightly longer fourth (8: 7); apical segment equal penultimate segment; width of face shorter than height of face and clypeus combined (13: 15); height of eyes 2.0 times width (14: 7); tentorial pits small, distance between tentorial pits 2.7 times distance from pit to eye margin (8: 3) (fig. 34); head in dorsal 
view with temple roundly narrowed posteriorly (fig. 33); ocelli small in a low triangle, the anterior tangent to the posterior ocelli touching anterior ocellus; POL equal times OOL and 2.0 times diameter of posterior ocellus (4: 2) (fig. 33 ); fons prominent with median longitudinal carina; face densely punctate; stemmaticum shiny, smooth; vertex, occiput and temple finely punctate.

Mesosoma.- Mesonotum as wide as head in dorsal view; mesoscutum with discrete punctures, punctures fading posteriorly; basal haft of scutellum with sharp discrete punctures becoming coriaceous posteriorly; scutellar sulcus dull and rugose (fig. 35); mesopleuron largely smooth, finely punctate anteriorly; propodeum with a short basal longitudinal carina, apical haft of propodeum rugose.

Wings.- Fore wing with radial vein $r$ arising after middle of pterostigma; vein $r$ and vein $2-$ SR curved not angled (fig. 39); metacarp 1.4 times length of pterostigma (28: 20); vein 1-
CU1, vein 2-CU1 and m-cu equal.

Legs.- Hind coxa shiny smooth, ratio of hind femur: tibia: tarsi 1-5 and basitarsus $=31: 34: 40$ : 16 ; inner hind tibial spurs about 0.6 times hind basitarsus (9: 16); fourth tarsal segment as long as fifth tarsal segment; inner side of hind basitarsus with strong setae (fig. 38).

Metasoma.- Horizontal surface of tergite 1 rugose, about 0.7 times as long as apical width (6: 9), slightly and roundly wider apically; median section of tergite 2 coriaceous, as long as horizontal surface of tergite 1 and shorter than tergite 3 (6: 7) (fig. 37); tergite 3 shiny and smooth.

Colour.- Body black; palpi white; scape yellow; flagellum yellowish brown basally; fore and middle legs yellow; hind coxa brown, yellow apically; trochanters and hind femur yellow; basal one third of hind tibia yellow, apical two third and hind tarsus brown; wing vein yellow; pterostigma and metacarp brown.

Male: unknown.

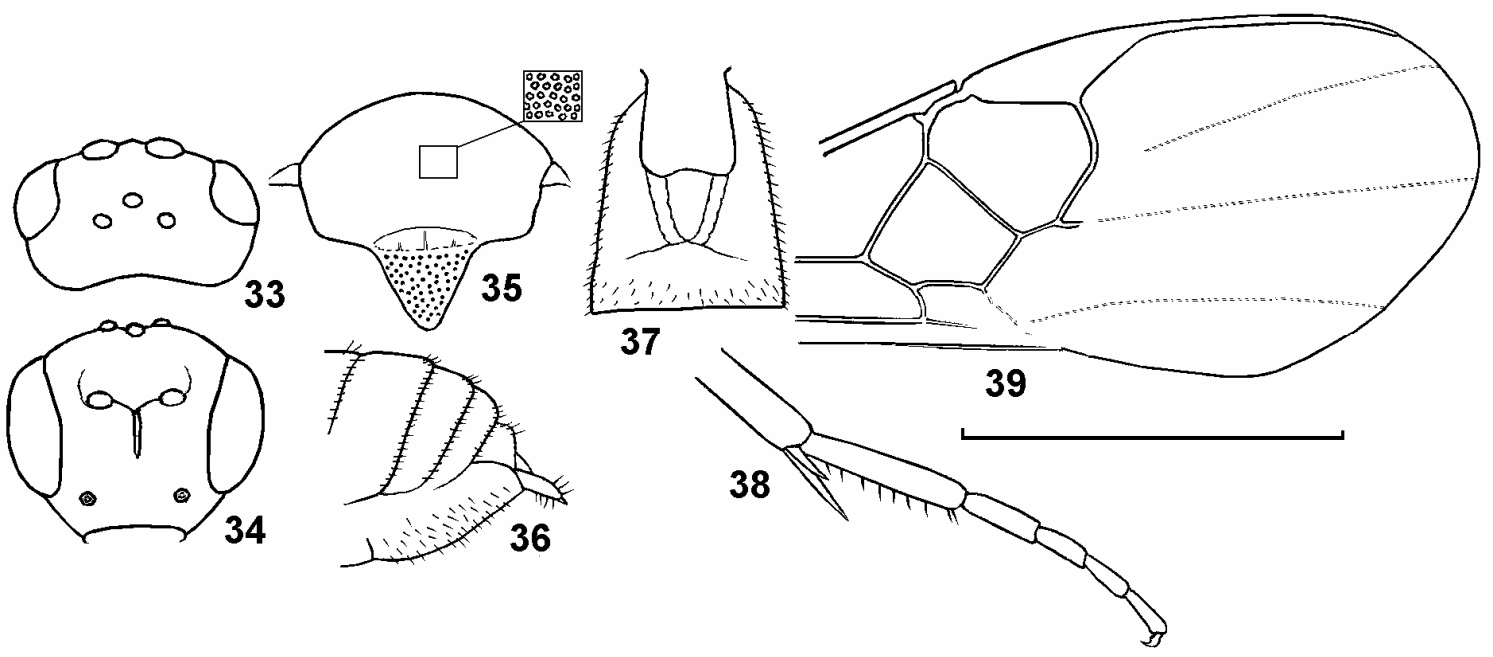

Figs 33-39. Protapanteles (Nyereria) yenthuyensis Long \& van Achterberg, sp. $\mathrm{n}$.

25. head, dorsal aspect; 26 . head, frontal aspect; 27. mesonotum; 28. apical metasomal segments, lateral aspect; 29. hind tarsus; 30. first-third tergites; 31. scutellum and metanotum, lateral aspect; 32. fore wing.

\section{REFERENCES}

1. Achterberg C. van, 1993: Zoologische Verhandelingen Leiden, 283: 1-189.

2. Austin A. D. \& Dangerfield P. C., 1992: Invertebrate Taxonomy, 6(1): 1-76.

3. Chen X. X., He J. H. \& Ma Y., 1994:
Entomotaxonomia (in Chinese with English summary), 16(2): 127-134.

4. Khuat Dang Long \& Belokobylskij S. A., 2003: Russian Entomological Journal, 12(4): 385-398.

5. Khuat Dang Long \& van Achterberg C., 2003: Zoologische Mededelingen Leiden, 
77(10): 221-227.

6. Khuat Dang Long, 2007: Journal of Biology, 29(2): 35-43.

7. Khuat Dang Long, 2007: Journal of Biology, 29(3): 25-31.

8. Khuat Dang Long, 2007: Proceedings of the $2^{\text {nd }}$ National Scientific Conference on Ecology and Biological Resources, 10: 140152.

9. Mason W. R. M., 1981: Memoirs of the Entomological Society of Canada. no. 115.

10. Nixon G. E. J., 1965: Bulletin of the British Museum (Natural History), Entomology series. Supplement, 2: 1-284.
11. Nixon G. E. J., 1967: Bulletin of the British Museum (Natural History), Entomology series, 21(1): 1-34.

12. Sathe T. V. \& Inamdar S. A.,1988: Journal of Advanced Zoology, 9(2): 128-131.

13. Sathe T.V. \& Inamdar S. A., 1991: Hexapoda (Insecta Indica), 3(1-2): 89-93.

14. Wilkinson D. S., 1932: Trans. R. ent. Soc. London, 80: 301-334.

15. Yu D. S., Achterberg K. van \& Horstmann K., 2005: Ichneumonoidea 2004 (Biological and taxonomical information), Taxapad Interactive Catalogue, Vancouver.

\title{
GHI NHẬN MỚI 2 GIỐNG VÀ 1 LOÀI CHO KHU HỆ, MÔ TẢ 5 LOÀI MỚI CHO KHOA HỌC THUỘC PHÂN HỌ ONG KÝ SINH MICROGASTRINAE (HYMENOPTERA: BRACONIDAE) Ở VIÊTT NAM
}

\author{
KHUẤT ĐĂNG LONG, C. VAN ACHTERBERG
}

\section{TÓM TÁT}

Phân họ ong ký sinh Microgastrinae là một trong những phân họ lớn với rất nhiều loài. Đến nay, ở Việt Nam chúng tôi mới thống kê được các loài thuộc 5 giống Apanteles Foerster, 1862; Cotesia Cameron, 1891; Fornicia Brullé, 1846; Microplitis Foerster, 1862; Wilkinsonellus Mason, 1981 [4, 5, 6, 7, 8]. Trong bài này, chúng tôi đưa ra ghi nhận mới cho khu hệ của Việt Nam 2 giống: Alloptilis Nixon, 1965 và Protapanteles Ashmead, 1898 (phân giống Nyereria Mason, 1981), 1 loài được ghi nhận mới cho khu hệ là Alloplitis guapo Nixon, 1965. Năm loài mới cho khoa học thuộc hai giống này được mô tả và minh họa, đó là Alloplitis albiventris Long \& van Achterberg, sp. n.; A. laevigaster Long \& van Achterberg, sp. n.; A. vietnamicus Long \& van Achterberg, sp. n.; Protapanteles (Nyereria) albicentrus Long \& van Achterberg, sp. n. và $P$. (Nyereria) yenthuyensis Long \& van Achterberg, sp. n..

\section{Giống Alloptilis Nixon, 1965}

Alloplitis Nixon, 1965. Bull. Br. Mus. (Natural History), Ent. Ser. Suppl., 2: 268.

Type-species: Alloplitis guapo Nixon, 1965.

Đặc điểm chẩn loại: thuộc tộc Microplitini. Nhìn bên sườn gờ trước của metanotum cách xa gờ sau của scutellum, phía đỉnh scutellum có vết lõm được viền bởi gờ nổi. Mesoscutum thường có rãnh lưng khá rõ ở nửa đỉnh và có gờ viền ở trên mấu ôm gốc cánh. Sườn bên pronotum có vết lõm rộng có nhiều khía nhăn. Đốt trung gian ít nhiều có khoang lõm với gờ viền rõ (hình 4,11,23), không có gờ dọc giữa. ống chân sau phình rộng ở đỉnh với ít lông cứng ngắn ở mặt ngoài (hình 5,15$)$, móng chân sau có một số răng như hình lược, cựa ống chân sau ngắn. Cánh trước có ô cánh marginal rộng thường có 4 cạnh, đôi khi 3 cạnh (hình 7, 14, 21). Cánh sau có gân cu-a lượn cong, rìa thùy cánh sau gần như thẳng và nhẳn. Tấm lưng bụng 1 ngắn hơi rộng ở đỉnh, tấm lưng bụng 2 và 3 là hai phần tương phản nhau, tấm lưng bụng 2 gần như hẹp về phía đỉnh, dài hơn tấm lưng bụng 3 và nhăn đều, tấm lưng bụng 3 nhẵn hoặc có khía dọc ở phần gốc (hình $8,13,22$ ). Mấu ôm bao máng đẻ trứng ngắn và nhẵn, bao máng đẻ trứng có lông măng ở sát phía đỉnh (hình 24).

Giống này hiện mới biết 3 loài đều thuộc khu hệ Đông Phương [15]. Dưới đây là khóa định loại cho bốn loài từ khu hệ họ ong ký sinh Braconidae của Việt Nam, trong số đó 1 loài là ghi nhận mới và 3 loài được mô tả là loài mới cho khoa học . 


\section{Khóa định loại 4 loài thuộc giống Alloptilis Nixon, 1965 ở Việt Nam}

1. Tấm lưng bụng 3 nhăn và dài bằng 0,5 lần tấm lưng bụng 2 , cánh trước có ô cánh submarginal nhỏ, đốt háng sau màu vàng. Alloplitis guapo Nixon, 1965

- Tấm lưng bụng 3 nhã̃n, dài hơn $2 / 3$ tấm lưng bụng 2 (hình 8,22 ), nếu ngắn hơn 0,5 lần tấm lưng bụng 2 khi đó tấm lưng bụng 2 nhẵn (hình 13) hoặc đốt háng sau đen hoặc nâu đen, cánh trước có ô cánh submarginal trung bình hoặc rộng (hình $7,14,21$ ), đốt háng sau nâu đen hoặc đen.

2. Tấm lưng bụng 3 ngắn hơn 1/2 tấm lưng bụng 2, tấm lưng bụng 2 nhẵn (hình 13), cựa ống chân sau dài gần bằng $1 / 2$ đốt bàn 1 chân sau (hình 15 )..... Alloplitis laevigaster sp. $\mathrm{n}$.

- Tấm lưng bụng 3 dài gần bằng 2/3 tấm lưng bụng 1 , tấm lưng bụng 2 nhăn (hình 8,22 ), cựa ống chân sau dài gần bằng hoặc ngắn hơn $1 / 3$ đốt bàn 1 chân sau (hình 5 ). 3

3. Cánh trước có ô cánh submarginal rộng (hình 21 ), đùi và ống chân sau màu vàng, bụng màu đen hoặc nâu đen Alloplitis vietnamicus sp. $\mathrm{n}$.

- Cánh trước có ô cánh submarginal trung bình (hình 7), đùi và ống chân sau màu đen, bụng màu nâu hai bên sườn bụng màu trắng Alloplitis albiventris sp. $\mathrm{n}$.

Alloplitis guapo Nixon, 1965 phân bố ở Philipin, ở Việt Nam loài này được tìm thấy ở VQG Cúc Phương, bẫy treo, $20^{\circ} 23^{\prime} 06 \mathrm{~N}-105^{\circ} 34^{\prime} 11 \mathrm{E}, 20-30$. VI.2002, K. Đ. Long.

\section{Alloplitis albiventris Long \& van Achterberg, sp. n. (hình 1-8)}

Mẫu vật. Holotype, †, Mic.570: VN, Hòa Bình (Yên Thủy), vườn quả (bẫy treo), 20²3’47N 105³6’14E, 01-10.IV.2002, K. Đ. Long.

Chiều dài: thân $2,7 \mathrm{~mm}$; cánh trước $2,7 \mathrm{~mm}$; râu đầu 3,0 mm. Vật chủ: chưa rõ.

Con đực: chưa rõ.

Nhận xét: Alloplitis albiventris sp. n. gần với loài A. guapo Nixon, 1965 của Philipin, nhưng loài này có đặc điểm khác ở chỗ: i)- tấm lưng bụng 3 nhẵn và dài gần bằng 0,9 lần tấm lưng bung 2, ii)- đốt trung gian có gờ nổi dọc gần hai bên sườn, iii)- giữa trán có gờ nổi và những nếp nhăn hình chữ $\mathrm{V}$ và iiii)- các tấm bụng $1+2+3$ có màu trắng.

Alloplitis laevigaster Long \& van Achterberg, sp. n. (hình 9-16)

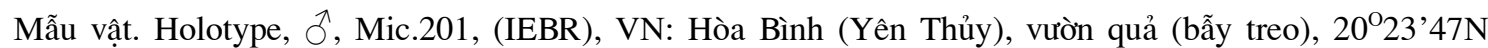
105³6’14E, 20-30.VII.2002, K. Đ. Long.

Chiều dài: thân 2,9 mm; cánh trước 3,2 mm; râu đầu 4,2 mm. Vật chủ: chưa rõ.

Con cái: chưa rõ.

Nhận xét: loài Alloplitis laevigaster sp. n. gần với loài A. completus Mason, 1981 của Malaysia nhưng có đặc điểm khác ở chỗ: i)- tấm bụng 1+2+3 đen, ii) - tấm lưng bụng 2 nhẵn và iii) - tấm lưng bụng 3 ngắn hơn 0,5 lần tấm lưng bung 2 . Loài này rất gần với loài $A$. typhon Nixon, 1965 của Philipin nhưng có đăc điểm khác ở chỗ: i)- tấm lưng bụng 3 ngắn hơn 0,5 lần tấm lưng bụng 2, ii)- ống chân sau màu nâu đen đến đen.

Alloplitis vietnamicus Long \& van Achterberg, sp. n. (hình 17-24)

Mẫu vật. Holotype, + , Mic. 939, (IEBR), VN: Vĩnh Phúc (Mê Linh), rừng thứ sinh (bẫy treo), 21 $23^{\circ} \mathrm{N}$

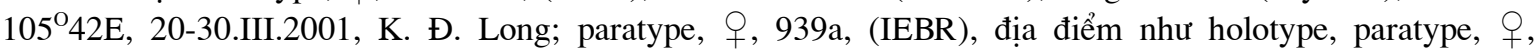
(RMNH), VN: Hà Giang (Vị Xuyên), vườn rừng 700m, (bẫy treo) 01-25.XI.2001, K. Đ. Long.

Chiều dài: thân $2,2 \mathrm{~mm}$; cánh trước $2,5 \mathrm{~mm}$; râu đầu 2,4mm. Vật chủ: chưa rõ.

Con đực: chưa rõ.

Nhận xét: loài Alloplitis vietnamicus sp.n. gần với loài A. guapo Nixon, 1965 của Philipin nhưng có đặc điểm khác ở chỗ: i)- tấm lưng bụng 3 nhẵn và dài hơn $1 / 2$ tấm lưng bụng 2 , ii)- chiều dài đốt râu sát đốt đỉnh ngắn hơn chiều rộng, iii)- cựa trong ống chân sau dài bằng $1 / 3$ đốt bàn 1 chân sau và iiii)- đốt trung gian không có gờ nổi dọc giữa (có nhiều gờ nổi). 


\section{Giống Protapanteles Ashmead,1898}

Protapanteles Ashmead,1898. Proc. Ent. Soc. Washington. 4: 155-171

Type-species (des.): Protapanteles ephyrae Ashmead, 1897 (= Apanteles paleacritae Riley)

Đặc điểm chẩn loại: giống Protapanteles (phân giống Nyereria Mason, 1981) [9] thuộc tộc Cotesiini. Máng đẻ trứng gần như bị mấu ôm (hypopygium) che kín, gần đỉnh của bao máng đẻ trứng có ít lông măng $(28,36)$. Tấm lưng bụng 1 bằng 1-2 lần chỗ rộng nhất (ở phân giống Nyereria) tấm lưng bụng 1 dài bằng 2 lần chỗ rộng nhất hoặc có cạnh gần như song song nhưng đều lượn tròn rõ ở đỉnh, tấm lưng bụng 2 có hai rãnh lõm chụm về phía đỉnh, hai rãnh lõm này tạo thành quầng gần như hình tam giác ngược (hình 30, 37), tấm lưng bụng 2 bằng $2 / 3$ chiều dài tấm lưng bụng 3 , quầng giữa rãnh lõm ở tấm lưng bụng 1 và 2 nhăn, tấm lưng bụng 3 thường nhẵn. Đốt trung gian nhã̃n hoặc nhăn mờ nhưng bóng, không có vết khoang lõm và không có gờ dọc giữa. Sườn bên pronotum tương tự như ở giống Cotesia Cameron. Cánh trước không có ô cánh submarginal, gân $\mathrm{r}$ và gân 2-SR dài và ít nhiều tạo góc gãy rõ. Thùy hậu cánh sau lồi có phủ lông măng. Đốt bàn 5 chân trước ở con cái thường có một lông măng cứng dài và uốn cong. Các loài thuộc giống này thường gặp ký sinh ở nhóm côn trùng thuộc họ Geometridae [9].

Giống này đã biết tới 212 loài thuộc khu hệ thế giới, phân giống Nyereria mới biết 19 loài, trong số đó 17 loài thuộc khu hệ của châu Phi, 1 loài thuộc khu hệ Đông Cổ Bắc còn khu hệ Đông Phương có 1 loài Protapanteles (Nyereria) ragheshri (Sathe, 1988) [12, 15]. Dưới đây là khóa định loại 2 loài mới cho khoa học từ khu hệ họ ong ký sinh Braconidae của Việt Nam.

\section{Khóa định loại 2 loài thuộc giống Protapanteles Ashmead, 1898 (phân giống Nyereria Mason, 1981) ở Việt Nam}

1. Ba mắt đơn rất thấp, tiếp tuyến rìa trước hai mắt đơn sau cắt hẳn vào giữa mắt đơn trước (hình 25 ); rãnh lõm trước scutellum có 7 gờ dọc rõ; scutellum có chấm lỗ tròn lớn và rời rạc giống như chấm lỗ ở giữa mesoscutum (hình 27); quầng giữa tấm lưng bụng 2 có khía dọc, phần còn lại hai bên tấm lưng bụng 2 và gốc tấm lưng bụng 3 có chấm lỗ nhỏ và thưa (hình 30); tấm lưng bụng 1 và háng sau màu vàng trắng (ở con đực, háng sau màu nâu đỏ) Protapanteles (Nyereria) albicentrus sp. $\mathrm{n}$.

- Ba mắt đơn cao hơn, tiếp tuyến rìa trước hai mắt đơn sau chỉ cắt rìa dưới mắt đơn trước (hình 33 ); rãnh lõm trước scutellum hẹp và nhăn; scutellum có chấm lỗ nhăn khác với chấm lỗ mờ và thưa ở giữa mesoscutum (hình 35); quầng giữa tấm lưng bụng 2 và tấm lưng bụng 3 nhẵn bóng (hình 37); tấm lưng bụng 1 và háng sau màu nâu đen hoặc đen. Protapanteles (Nyereria) yenthuyensis sp. $\mathrm{n}$.

Protapanteles (Nyereria) albicentrus Long \& van Achterberg, sp. n. (hình 25-32)

Mẫu vật. Holotype, †, (IEBR), Apan.740, VN: Hà Tĩnh (Hương Sơn), rừng, (bẫy treo), 2059’N 105 $55^{\prime}$ 'E, 22.VI- 08.VII.2001, AMNH, K. Long. Paratype đ̃, (IEBR), Mic. 1044, địa điểm như holotype, rừng 900m, (bẫy treo), 05.V.1998, AMNH, K. Long; paratype, + , (RMNH) Mic.1023, VN: Hà Tĩnh (Hương Sơn), rừng 900m, (bẫy treo), 18²2’ N - 106²13’E, 20-28.IV.1998, AMNH, K. Long.

Chiều dài: thân 3,4 mm; cánh trước 3,6 mm; râu đầu 3,7 mm.Vật chủ: chưa rõ.

Protapanteles (Nyereria) yenthuyensis Long \& van Achterberg, sp. n. (hình 33-39)

Mẫu vật. Holotype, ㅇ, (IEBR), Mic.918: VN, Hòa Bình (Yên Thủy), (bẫy treo), rừng, 20²8'N 105³4'E, 20-30.X.2003, K. Đ. Long.

Chiều dài: thân $2.3 \mathrm{~mm}$; cánh trước $2,6 \mathrm{~mm}$; râu đầu $2,5 \mathrm{~mm}$.Vật chủ: chưa rõ.

Con đực: chưa rõ.

Ngày nhận bài: 15-2-2008 